\title{
Dexamethasone therapy versus surgery for chronic subdural haematoma (DECSA trial): study protocol for a randomised controlled trial
}

Ishita P. Miah ${ }^{1 *}$ D, Dana C. Holl², Wilco C. Peul ${ }^{1,4,5}$, Robert Walchenbach¹, Nyika Kruyt ${ }^{4}$, Karlijn de Laat ${ }^{5}$, Radboud W. Koot ${ }^{4}$, Victor Volovici ${ }^{2}$, Clemens M. F. Dirven ${ }^{2}$, Fop van Kooten ${ }^{2}$, Kuan H. Kho ${ }^{6}$, Heleen M. den Hertog ${ }^{7}$, Joukje van der Naaltt, Bram Jacobs ${ }^{8}$, Rob J. M. Groen ${ }^{8}$, Hester F. Lingsma ${ }^{3}$, Ruben Dammers ${ }^{2}$, Korné Jellema ${ }^{1 \dagger}$, Niels A. van der Gaag ${ }^{1,4,5+}$ on behalf of the Dutch Subdural Hematoma Research Group (DSHR)

\begin{abstract}
Background: Chronic subdural haematoma (CSDH) is a common neurological disease with a rapidly rising incidence due to increasing age and widespread use of anticoagulants. Surgical intervention by burr-hole craniotomy (BHC) is the current standard practice for symptomatic patients, but associated with complications, a recurrence rate of up to $30 \%$ and increased mortality. Dexamethasone (DXM) therapy is, therefore, used as a non-surgical alternative but considered to achieve a lower success rate. Furthermore, the benefit of DXM therapy appears much more deliberate than the immediate relief from BHC. Lack of evidence and clinical equipoise among caregivers prompts the need for a head-to-head randomised controlled trial. The objective of this study is to compare the effect of primary DXM therapy versus primary BHC on functional outcome and cost-effectiveness in symptomatic patients with CSDH.

Methods/Design: This study is a prospective, multicentre, randomised controlled trial (RCT). Consecutive patients with a CSDH with a Markwalder Grading Scale (MGS) grade 1 to 3 will be randomised to treatment with DXM or BHC. The DXM treatment scheme will be $16 \mathrm{mg}$ DXM per day (8 mg twice daily, days 1 to 4 ) which is then halved every 3 days until a dosage of $0.5 \mathrm{mg}$ a day on day 19 and stopped on day 20. If the treatment response is insufficient (i.e. persistent or progressive symptomatology due to insufficient haematoma resolution), additional surgery can be performed. The primary outcomes are the functional outcome by means of the modified Rankin Scale (mRS) score at 3 months and cost-effectiveness at 12 months. Secondary outcomes are quality of life at 3 and 12 months using the Short Form Health Survey (SF-36) and Quality of Life after Brain Injury Overall Scale (QOLIBRI), haematoma thickness after 2 weeks on follow-up computed tomography (CT), haematoma recurrence during the first 12 months, complications and drugrelated adverse events, failure of therapy within 12 months after randomisation and requiring intervention, mortality during the first 3 and 12 months, duration of hospital stay and overall healthcare and productivity costs. To test noninferiority of DXM therapy compared to BHC, 210 patients in each treatment arm are required (assumed adjusted common odds ratio DXM compared to BHC 1.15, limit for inferiority < 0.9). The aim is to include a total of 420 patients in 3 years with an enrolment rate of $60 \%$.

(Continued on next page)
\end{abstract}

\footnotetext{
* Correspondence: i.miah@haaglandenmc.nl

†Korné Jellema and Niels A. van der Gaag contributed equally to this work.

'Department of Neurology and Neurosurgery, Haaglanden Medical Centre

(HMC), Lijnbaan 32, 2512 VA The Hague, The Netherlands

Full list of author information is available at the end of the article
}

(c) The Author(s). 2018 Open Access This article is distributed under the terms of the Creative Commons Attribution 4.0 International License (http://creativecommons.org/licenses/by/4.0/), which permits unrestricted use, distribution, and reproduction in any medium, provided you give appropriate credit to the original author(s) and the source, provide a link to the Creative Commons license, and indicate if changes were made. The Creative Commons Public Domain Dedication waiver (http://creativecommons.org/publicdomain/zero/1.0/) applies to the data made available in this article, unless otherwise stated. 
(Continued from previous page)

Discussion: The present study should demonstrate whether treatment with DXM is as effective as BHC on functional outcome, at lower costs.

Trial registration: EUCTR 2015-001563-39. Date of registration: 29 March 2015.

Keywords: Dexamethasone, DXM, Chronic subdural haematoma, CSDH, Burr-hole craniostomy, BHC

\section{Background}

A chronic subdural haematoma (CSDH) is a common neurological disease with a rapidly rising prevalence due to increasing age and the widespread use of anticoagulants [1-4]. It generally affects the elderly population and patients with coagulopathy, who often have co-existing medical diseases $[1,5]$. The estimated incidence in Western countries is 8.1 per 100,000 per year in patients aged 65 years or older [6], but increases to $58 / 100,000 /$ year for those aged 70 years or older $[1,7]$.

Surgical intervention by burr-hole craniotomy (BHC) followed by subdural drainage is the mainstay treatment in symptomatic patients with a $\operatorname{CSDH}[8,9]$, which leads to a favourable functional outcome in $84 \%$ of patients [10]. However, despite the optimisation of techniques surgery is still associated with relevant complications, recurrence rates up to $30 \%$, and considerable mortality [8-12]. In addition, especially advanced age and the presence of comorbidities could render patients ineligible for BHC.

Dexamethasone (DXM) therapy has been proposed as an alternative, non-operative or adjuvant treatment modality and might have the potential to block the anti-inflammatory changes in the formation of the haematoma and can specifically impede the formation of neo-membranes and neo-capillaries by its powerful inhibition of inflammatory mediators [13, 14]. Therefore, DXM is administered routinely in various institutions.

To date, only three retrospective and one prospective study have compared the clinical effect of DXM to $\mathrm{BHC}$ in $\mathrm{CSDH}$ patients [15]. To date, no randomised trials have been published that compare both treatments. Therefore, we designed the DECSA trial: a randomised controlled, multicentre trial to evaluate the non-inferiority of primary DXM compared to primary $\mathrm{BHC}$ on functional outcome and cost-effectiveness in patients with symptomatic CSDH.

\section{Methods/Design}

\section{Trial design}

This is a prospective, multicentre, open-label, randomised controlled trial (RCT) with a blinded endpoint (PROBE design) assessment [16]. Eligible patients are randomised to DXM therapy (the intervention arm) or BHC (control arm; see Additional file 1 for SPIRIT check-list).

\section{Primary study objective}

The primary objective is to evaluate the non-inferiority of primary DXM therapy versus primary BHC on functional outcome as expressed by modified Rankin Scale (mRS) score (Table 1) at 3 months and cost-effectiveness at 12 months in patients with symptomatic CSDH.

\section{Secondary objectives}

The secondary objectives of the study are functional and clinical outcome, expressed by mRS and Markwalder Grading Scale (MGS) scores (Table 2), respectively, at discharge, at 2 weeks, 3, 6 and 12 months and Glasgow Outcome Scale-Extended (GOSE) score (Table 3) at 3 months. Furthermore, assessment of quality of life using the Short Form - 36 Health Survey (SF-36) and Quality of Life after Brain Injury Overall Scale (QOLIBRI) will take place at 3 and 12 months and healthcare and productivity costs at 3 and 12 months. Haematoma thickness will be evaluated after 2 weeks on follow-up computed tomography $(\mathrm{CT})$. Mortality will be evaluated during the first 3 and 12 months. During the total follow-up period of 12 months we will also evaluate haematoma recurrence, complications and drug-related adverse events, failure of therapy after randomisation and requiring intervention, duration of hospital stay and overall healthcare and productivity costs.

\section{Study setting and participants}

Patients will be recruited for the study from the emergency department, neurological or neurosurgical outpatient clinic or ward or through referral from general hospitals of the seven participating Dutch neurosurgical hospitals. The seven participating neurosurgical hospitals are Haaglanden Medical Centre (HMC) The Hague, Haga Teaching Hospital The Hague, Leiden University Medical Centre (LUMC) in Leiden, Medisch Spectrum Twente (MST) Enschede, Erasmus Medical Centre (EMC) Rotterdam, Isala Hospital Zwolle and University Medical Centre Groningen (UMCG). The study is open to additional participating neurosurgical centres.

\section{Inclusion criteria}

Eligible patients must be 18 years or older and meet all of the following criteria: 
Table 1 Modified Rankin Scale (mRS)

\begin{tabular}{ll}
\hline Score & Functional status \\
\hline 0 & No symptoms \\
1 & $\begin{array}{l}\text { No significant disability. Able to carry out } \\
\text { all usual activities despite some symptoms } \\
2\end{array}$ \\
$\begin{array}{l}\text { Slight disability. Able to look after own } \\
\text { affairs without assistance, but unable to } \\
\text { carry out all previous activities }\end{array}$ \\
Moderate disability. Requires some help, \\
but able to walk unassisted \\
Moderately severe disability. Unable to \\
attend to own bodily needs without \\
assistance, and unable to walk unassisted \\
Severe disability. Requires constant nursing \\
care and attention, bedridden, incontinent \\
Dead
\end{tabular}

1) The presence of a newly diagnosed CSDH, defined as an isodense or hypodense haematoma in the subdural space on cranial computed tomography (CT) scan. Hyperdense components may be present but must compromise less than one third of the haematoma

2) Clinical symptoms must be explained by the CSDH

3) The patient is eligible for BHC and DXM based on clinical symptoms and radiological appearance of $\mathrm{CSDH}$

4) MGS grade 1-3.

The MGS is a validated grading system (score $0-4$, see Table 2) for the severity of neurological symptoms and is used to classify the neurological condition for CSDH patients [17]

\section{Exclusion criteria}

Exclusion criteria are:

1) MGS grade 0 or 4

2) An acute subdural haematoma

Table 2 Markwalder Grading Scale

\begin{tabular}{ll}
\hline Score & Clinical status \\
\hline 0 & Patient neurological normal \\
1 & $\begin{array}{l}\text { Patient alert and oriented; mild symptoms such as headache; } \\
\text { absent or mild neurological deficit such as reflex asymmetry }\end{array}$ \\
2 & $\begin{array}{l}\text { Patient drowsy (defined as Glasgow Coma Scale (GCS) score: } \\
13-14) \text { or disoriented with variable neurological deficit, such } \\
\text { as hemiparesis }\end{array}$ \\
3 & $\begin{array}{l}\text { Patient stuporous (defined as GCS 9-12) but responding } \\
\text { appropriately to noxious stimuli; severe focal signs such } \\
\text { as hemiplegia }\end{array}$ \\
4 & $\begin{array}{l}\text { Patient comatose (GCS } 8 \text { or lower) with absent motor } \\
\text { responses to painful stimuli; decerebrate or decorticate posturing }\end{array}$
\end{tabular}

Table 3 Glasgow Outcome Scale-Extended

\begin{tabular}{ll}
\hline Score & Category \\
\hline 1 & Death \\
2 & Vegetative state \\
3 & Severe disability, lower \\
4 & Severe disability, upper \\
5 & Moderate disability, lower \\
6 & Moderate disability, upper \\
7 & Good recovery, lower \\
8 & Good recovery, upper \\
\hline
\end{tabular}

3) The presence of a minimal CSDH on cranial CT which is technically not drainable by $\mathrm{BHC}$

4) Pregnancy

5) Cerebrospinal fluid shunt in situ (e.g. ventriculoperitoneal shunt)

6) Known hypersensitivity to DXM

7) Known ulceration in the gastro-intestinal tract

8) Poorly regulated diabetes mellitus (DM) defined as a glycosylated haemoglobin (HbA1C) value $>8 \%$ $(64 \mathrm{mmol} / \mathrm{mol})$

9) Clinical suspicion of an acute systemic infection (fever, leucocytosis, elevated C-reactive protein)

10) History of gastro-intestinal bleeding

11) Glaucoma

12) Previous history of severe affective disorders (i.e. psychosis).

\section{Participant timeline}

The time schedule in Fig. 1 describes all study processes, assessments and interventions. The flow diagram (Fig. 2) displays the main study procedures, including follow-up evaluations.

In summary, study patients will be evaluated at presentation (baseline), during their hospital stay, at discharge and during the follow-up period at 2 weeks, 3 months, 6 months and 12 months.

At 2 weeks (after initiation of the study treatment) patients will be evaluated by neurological examination combined with a follow-up CT scan at the outpatient clinic or ward and at 3 months at the outpatient clinic. A mRS-certified research nurse, blinded for treatment allocation, will evaluate the primary outcome (mRS score) at 3 months by phone. At 3 and 12 months, patients will receive questionnaires on quality of life. Additionally, an evaluation of mRS score will take place by phone at 3, 6 and 12 months. Healthcare and productivity costs will be evaluated at 3 and 12 months. We expect to complete patient inclusion in 3 years. The estimated duration of the study (including follow-up) will be 4 years. 


\begin{tabular}{|c|c|c|c|c|c|c|c|c|c|}
\hline Study procedure & $\begin{array}{l}\text { Initial } \\
\text { screening }\end{array}$ & $\begin{array}{l}\text { During } \\
\text { admission }\end{array}$ & $\begin{array}{l}\text { Day of } \\
\text { surgery }\end{array}$ & $\begin{array}{l}\text { Day 1-2 } \\
\text { post } \\
\text { surgery }\end{array}$ & $\begin{array}{l}\text { At } \\
\text { discharge }\end{array}$ & $\begin{array}{l}\mathrm{FU}(2 \\
\text { weeks) }\end{array}$ & $\begin{array}{l}\text { FU (3 } \\
\text { months) }\end{array}$ & $\begin{array}{l}\mathrm{FU} \text { (6 } \\
\text { months) }\end{array}$ & $\begin{array}{l}\text { FU (12 } \\
\text { months) }\end{array}$ \\
\hline Medical history & $\mathrm{X}$ & & & & & & & & \\
\hline Demographics & $\mathrm{x}$ & & & & & & & & \\
\hline $\begin{array}{l}\text { Concomitant } \\
\text { medications }\end{array}$ & $\mathrm{x}$ & $\mathrm{x}$ & $\mathrm{x}$ & & $\mathrm{x}$ & & & & \\
\hline $\begin{array}{l}\text { Neurological } \\
\text { examination }\end{array}$ & $x$ & $\mathrm{X}\left(\mathrm{OD}^{1}\right)$ & $\mathrm{X}$ & & $x$ & $\mathrm{x}^{2}$ & $x^{3}$ & & \\
\hline Vital parameters & $\mathrm{X}$ & $X\left(O^{4}\right)$ & $\mathrm{X}$ & & $x$ & & & & \\
\hline CT - scan & $x$ & & & & & $x^{5}$ & & & \\
\hline $\begin{array}{l}\text { Laboratory } \\
\text { evaluation }\end{array}$ & $\mathrm{x}$ & $x^{6}$ & $x^{7}$ & $x^{7}$ & & & & & \\
\hline $\begin{array}{l}\text { Glucose } \\
\text { monitoring }\end{array}$ & $\mathrm{X}$ & $x^{8}$ & & & & & & & \\
\hline $\begin{array}{l}\text { Informed } \\
\text { consent }\end{array}$ & $x^{9}$ & & & & & & & & \\
\hline MGS & $\mathrm{X}$ & & $\mathrm{X}$ & & $\mathrm{X}$ & $\mathrm{X}$ & $\mathrm{X}$ & $\mathrm{X}^{11}$ & $X^{11}$ \\
\hline mRS & $\mathrm{X}$ & & & & $\mathrm{X}$ & $\mathrm{X}$ & $X^{10}$ & $\mathrm{X}^{11}$ & $X^{11}$ \\
\hline GOSE & & & & & & & $X^{10}$ & & \\
\hline $\begin{array}{l}\text { Quality of life } \\
\text { (SF-36, } \\
\text { QOLIBRI) }\end{array}$ & $\overline{X^{12}}$ & & & & & & $\mathrm{X}^{12}$ & & $\mathrm{X}^{12}$ \\
\hline Costs & $\mathrm{X}^{10}$ & & & & & & $\mathrm{X}^{12}$ & & $\mathrm{X}^{12}$ \\
\hline Complications & & $\mathrm{X}$ & & & $\mathrm{X}$ & $\mathrm{X}$ & $\mathrm{X}$ & $\mathrm{X}$ & $\mathrm{x}$ \\
\hline Adverse events & & $\mathrm{X}$ & & & $\mathrm{x}$ & $\mathrm{X}$ & $\mathrm{X}$ & $\mathrm{X}$ & $\mathrm{x}$ \\
\hline $\begin{array}{l}\text { Serious adverse } \\
\text { events }\end{array}$ & & $\mathrm{X}$ & & & $x$ & $\mathrm{X}$ & $\mathrm{X}$ & $\mathrm{X}$ & $x$ \\
\hline Mortality & & $\mathrm{x}$ & & & $\mathrm{X}$ & $\mathrm{X}$ & $x$ & $\mathrm{X}$ & $x$ \\
\hline
\end{tabular}

Fig. 1 Time schedule of study procedures

\section{Interventions}

\section{Investigational treatment}

Patients in the intervention arm will receive DXM in a daily dosage of $16 \mathrm{mg}$ ( $8 \mathrm{mg}$ every $12 \mathrm{~h}$ ) on days 1 to 4 . Thereafter, DXM will be tapered by half every 3 days (see Table 4 for the DXM dosing scheme) until a dosage of $0.5 \mathrm{mg}$ a day on day 19 and stopped on day 20. DXM is administered orally in tablets or intravenously when oral administration is not possible. If the patient improves on DXM therapy (defined by $\geq 1$ point decrease in MGS score) during the first 2 weeks, the treatment will be continued until day 19. During DXM treatment a proton pump inhibitor (pantoprazole, $40 \mathrm{mg}$ daily) is administered as prophylaxis.

DXM therapy can be discontinued for the following reasons: (1) no improvement of the clinical condition, defined as an unchanged MGS score 2 weeks after initiation of DXM therapy with unchanged or increased haematoma on the follow-up CT at 2 weeks, (2) clinical deterioration, defined by as $\geq 1$ point increase in MGS score, at any time after initiation of DXM treatment, (3) the occurrence of severe, relevant DXM-related side effects or complications (i.e. uncontrollable hyperglycaemia, gastro-intestinal bleeding or psychiatric symptoms), (4) pre-study complement discontinuation of DXM therapy is primarily left to the discretion of the treating physician and is recommended in case of: persistence of moderate to severe neurological symptoms (MGS grade 2-3) in combination with the presence of relevant severe, current comorbidities (i.e. an infection, metabolic deterioration) which could interfere with the expected recovery, and a surgical intervention could be beneficial and the safest option for patient recovery.

In any case of pre-study discontinuation of DXM therapy, the reason for this is documented. Whenever DXM is discontinued, a cross-over to the reference treatment $(\mathrm{BHC})$ can occur depending on the remaining symptoms, which is the local standard of care in the participating hospitals.

\section{Reference treatment}

Patients randomised to the reference treatment arm are operated on preferably within the first 7 days, depending on anticoagulant or antithrombotic therapy use, severity of symptoms and discretion of the treating physician. Surgery will take place through BHC followed by insertion of a subdural drain for 2 days in line with the standard protocols in each participating hospital. Antibiotic prophylaxis is administered preoperatively. Either general or local anaesthesia will be applied. One or two 14-mm 


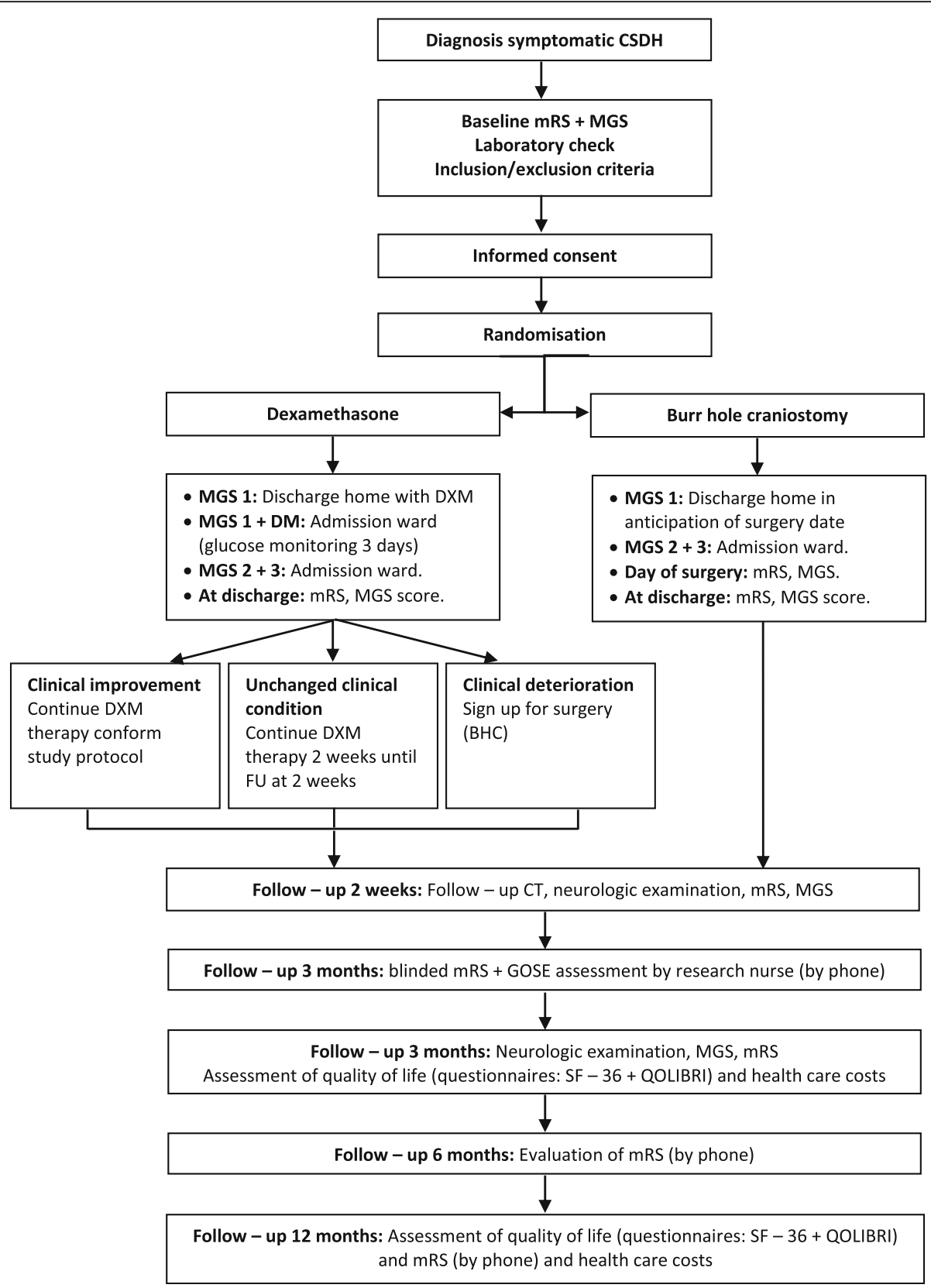

Fig. 2 Flow diagram of main study procedures

Table 4 Dexamethasone (DXM) dosing scheme

\begin{tabular}{ll}
\hline Day & DXM dosage \\
\hline $1-4$ & $8 \mathrm{mg}$ every $12 \mathrm{~h}$ \\
$5-7$ & $4 \mathrm{mg}$ every $12 \mathrm{~h}$ \\
$8-10$ & $2 \mathrm{mg}$ every $12 \mathrm{~h}$ \\
$11-13$ & $1 \mathrm{mg}$ every $12 \mathrm{~h}$ \\
$14-16$ & $0.5 \mathrm{mg}$ every $12 \mathrm{~h}$ \\
$17-19$ & $0.5 \mathrm{mg}$ per day \\
20 & Stop \\
\hline
\end{tabular}

burr holes, depending on the surgeon's discretion, are drilled over the maximum width of the haematoma. The dura mater is opened with a cruciate incision and coagulated with bipolar diathermy. The subdural collection is washed out with warm Ringer's lactate saline, with or without a catheter. The subdural outer and inner membrane loculations, if present, can be disrupted when easily accessible via the burr holes. Whenever the saline has dispersed sufficiently a subdural drain is placed and the wound is closed. 
Reoperation can be indicated when neurological deficits do not resolve, deteriorate or recur within the follow-up duration. Treatment options consist of redo burr-hole evacuation, if necessary, through another additional hole, percutaneous aspiration, craniotomy, or craniectomy.

\section{Concomitant care}

All included patients will otherwise receive routine standard of care. Patients with mild neurological deficits (MGS grade 1) on admission can be discharged home in anticipation of the planned BHC or awaiting the effect of DXM therapy. However, in MGS grade 1 patients with known diabetes mellitus (DM) with $\mathrm{HbA} 1 \mathrm{C}<64 \mathrm{mmol} /$ mol randomised for DXM therapy, monitoring for blood glucose levels is necessary during the first 3 days after treatment initiation. Glucose monitoring can take place clinically during admission or if possible at the nursing home. Patients with MGS grade 2-3 (in either arm) remain in hospital until the treating physician judges the clinical situation safe for discharge.

During admission neurological investigations and vital parameters are recorded daily. Low-molecular-weight heparin (LMWH) will be applied in both patient groups as thrombosis prophylaxis if the patient is not optimally mobile. Patients will receive physiotherapy, speech therapy or rehabilitation consultation if deemed necessary.

Anticoagulant or antithrombotic therapy Oral anticoagulant or antithrombotic therapy will be discontinued in both study arms from the moment of randomisation to prevent haematoma growth and to avoid interference with planned surgery. In case of vitamin $\mathrm{K}$ antagonist (VKA) therapy the international normalised ratio (INR) is corrected to $\leq 1.5$ through the administration of vitamin $\mathrm{K}$ and/or prothrombin complex concentrate (PCC), as is the current practice. For patients using platelet-aggregation-inhibitor therapy, surgery is preferably planned 7 days after discontinuation of therapy, if allowed by the clinical condition. At the discretion of the surgeon, earlier intervention is allowed if deemed clinically necessary. The reason for early surgery has to be recorded in the case report form (CRF). Non-vitamin-K oral anticoagulants (NOACs) are discontinued at least 1 day prior to surgery.

Any anticoagulant or antithrombotic therapy can be resumed 2 weeks after the initiation of DXM therapy or surgery following a follow-up CT without signs of CSDH recurrence, recent-onset haematoma or unchanged mass effect with midline shift compared to the initial CT at randomisation. Partial resolution of $\mathrm{CSDH}$ at this stage without recent haematoma is not a contraindication for resumption. For absolute indications (e.g. mechanic cardiac valve) earlier resumption or bridging of therapy within these 14 days is allowed. Any reason for early resumption has to be recorded in the CRF. Subgroup analyses will be performed to evaluate the effect of anticoagulant therapy in both groups.

\section{Outcomes}

\section{Primary outcome measures}

The primary endpoints are the functional outcome, expressed by mRS, at 3 months after start of study treatment and cost-effectiveness at 12 months.

\section{Secondary outcome measures}

Secondary outcomes include: functional and clinical outcome, expressed by mRS and MGS scores, respectively, at discharge, at 2 weeks, at 3, 6 and 12 months after start of study treatment. We will also determine a utility-weighted mRS (UW-mRS) at 3 months. The GOSE score will be assessed at 3 months, quality of life (expressed by SF-36 and QOLIBRI) at 3 and 12 months, cost-effectiveness at 3 and 12 months and haematoma thickness at 2 weeks. During the first 12 months, we will evaluate haematoma recurrence (defined as recurrence of symptoms and neurological signs after initial improvement with persistence, recurrence or increase of CSDH on follow-up CT), failure of therapy after randomisation and requiring intervention, complications and drug-related adverse events, duration of hospital stay and healthcare and productivity costs in both patient groups. Finally, we will evaluate mortality during the first 3 and 12 months.

\section{Randomisation}

Patients are randomised in a 1:1 allocation ratio stratified for study site by their treating physician. Stratified block-randomisation is done by using a computer randomisation algorithm to generate balanced random samples (Castor EDC, Ciwit B.V., Amsterdam, The Netherlands).

\section{Sample size}

This RCT is designed as a non-inferiority study. The sample size for showing non-inferiority is calculated based on a simulation programme in $\mathrm{R}$ statistical software for power for ordinal regression. We aim to include 420 patients. This sample size yields a power of $90 \%$, assuming that the true effect of DXM is an odds ratio 1.15 for a better functional outcome on the mRS, and the limit for inferiority is an odds ratio $<0.9$.

\section{Data collection}

All patient data is collected in the electronic data capture software Castor EDC (Ciwit B.V., Amsterdam, The Netherlands). This software allows built-in logical checks and validations to promote data quality. Data entry is performed locally by trained research nurses and physicians. No patient-identifying information is collected. 


\section{Data analysis}

The primary effect parameter (and all other comparisons of the treatment arms) will be performed on all randomised subjects according to the intention-to-treat (ITT) principle. A sensitivity analysis is performed for the primary outcome measure in a per-protocol fashion, defined as patients in the ITT population receiving treatment as randomised without protocol violation.

The primary effect parameter will be the adjusted common odds ratio (acOR) for a shift in the direction of a better outcome on the mRS at 3 months with $95 \%$ confidence interval, estimated with multivariable ordinal logistic regression with adjustment for important prognostic baseline variables. This analysis is becoming the standard for ordinal functional outcomes in neurology and neurosurgery, supported by evidence for its maximisation of statistical power while maintaining interpretability. Missing data in baseline characteristics will be imputed using multiple imputation $(n=10)$ based on the outcome and relevant baseline covariates using the 'Multivariate Imputation by Chained Equations' (MICE) algorithm. Patients with missing primary outcome will be excluded but every effort will be made to obtain follow-up. To accept the null hypothesis (H0) of non-inferiority the lower 95\% confidence limit of the odds ratio for a better functional outcome on the mRS of DXM versus surgery should be equal to or above 0.9.

Furthermore, we will perform an extensive economic evaluation of DXM versus surgery for patients with a $\mathrm{CSDH}$. The economic evaluation will be performed according to the Dutch guidelines, using a societal perspective. The timeframe will be 12 months to take all relevant costs and effects into account. The primary effect measure for the economic evaluation will be functional status (mRS). Secondary outcome measures for the cost-effectiveness analyses (CEA) will be mortality and quality-adjusted life year (QALY), based on the 12-month SF-36 and QOLIBRI summary scores. The cost-effectiveness will be assessed by calculating the incremental cost-effectiveness ratio (ICER), defined as the difference in costs, divided by the average change in effectiveness of DXM versus surgery in CSDH patients. The cost-effectiveness analysis will use the mRS as effect measure and the cost-utility analysis will use the QALY as effect measure.

Uncertainty around this ratio will be presented using confidence eclipses on the cost-effectiveness plane and acceptability curves. We will perform a sensitivity analysis to assess the robustness of the results to changes in costs and effectiveness parameters. Due to the short time horizon, no discounting for costs and effects will be used (see Additional file 2 for statistical analysis plan).

For secondary endpoint parameters, Kaplan-Meier and Cox regression analysis will be used for mortality comparisons between the treatment arms, binary logistic regression for complications and failure of therapy, and a linear regression to evaluate quality of life. A $p$ value of less than 0.05 will be used to indicate statistical significance. For all analyses, R statistical software will be used.

\section{Study monitoring Data monitoring}

The coordinating investigator will visit study centres every 3 months to discuss any issues and check on conduct of the study. Prior to recruitment, the field team (physicians) will receive information and instructions on the objectives of the study, methods and processes of the study. CRF data will be monitored by an independent external expert at regular intervals throughout the study to verify adherence to the protocol and data completeness, consistency and accuracy.

\section{Data Safety Monitoring Board (DSMB)}

In order to increase the safety of the intervention the trial will be monitored by an independent Data Safety Monitoring Board (DSMB). The DSMB will work in accordance with a dedicated charter and will follow processes recommended by the DAMOCLES Statement. The DSMB will be chaired by a neurosurgeon, and include a neurologist and an independent methodologist/ statistician. The DSMB will meet at least annually or after inclusion of the next 150 patients (whichever comes first). With respect to study safety and efficacy, interim analyses of major endpoints (including serious adverse events believed to be due to treatment) are performed after 150 and 300 patients have completed their follow-up evaluation. In addition, the DSMB will review the study logistics/trial conduct in terms of: assessment of compliance with the study protocol (including adherence to inclusion and exclusion criteria) and monitor data quality (completeness), time to start of the procedure (DXM/surgery), cross-overs, occurrence and listing/registration of (serious) adverse events, by centre and by treatment arm.

\section{Adverse events (AEs) and serious adverse events (SAEs)}

Adverse events are defined as any undesirable event occurring to a patient during the study, whether or not considered related to DXM therapy or surgery. All adverse events reported spontaneously by the patient or observed by the investigator or staff will be recorded. A SAE is any untoward medical occurrence or effect that results in death; is life-threatening (at the time of the event); requires hospitalisation or prolongation of existing inpatients' hospitalisation; results in persistent or significant disability or incapacity or any other important medical event that did not result in any of the outcomes listed above due to medical or surgical intervention, but could have been based upon appropriate judgement by 
the investigator. SAEs are reported by the investigators in participating centres to the coordinating investigator. SAEs will be reported through the web portal ToetsingOnline to the accredited Medical Ethics Committee that approved the protocol.

\section{Interim analysis}

Interim analyses of major endpoints (including serious adverse events believed to be due to treatment) are performed after 150 and 300 patients have completed their follow-up evaluation.

\section{Dissemination of results}

Trial results will be published in an international journal, communicated to neurological and neurosurgical associations and presented at (inter)national congresses.

\section{Discussion}

General guidelines defining the preferred treatment for $\mathrm{CSDH}$ are lacking, but worldwide, surgery is the current standard practice. Various hospitals, however, apply DXM as an alternative treatment modality or as an adjunctive therapy prior to surgery. To date, no head-to-head trial comparing the two modalities in a well-defined cohort of patients has been performed to our knowledge. The competing benefit of either treatment is, therefore, not clear.

CSDH development occurs likely due to (mild) traumatic brain injury causing a tear in the dural border cell layer which leads to extravasation of cerebrospinal fluid and blood in the subdural space. At a point neurological deficits arise because of a mass effect due to liquefaction and progressive enlargement of an initially small haematoma. The rationale behind corticosteroid therapy is based on results of previous experimental work that postulates an inflammatory response to be responsible for the haematoma enlargement [13, 14, 18-23]. Accumulated blood in the subdural space, in particular erythrocyte breakdown products, incites an inflammatory reaction that results in the deposition of fibrin and formation of subdural neo-membranes with in-growth of neo-capillaries. These neo-membranes are vulnerable structures with high vascularisation of the outer layer and are prone to rupture and bleed. Furthermore, it is also believed that the outer layer of the neo-membrane contains a high content of plasminogen and plasminogen activator, which cause an enzymatic fibrinolysis and liquefaction of the initial blood clot in the inner haematoma. This situation finally results in frequent effusions of plasma or rebleeding from the neo-membranes into the subdural collection. Hence, a cascade of inflammation, impaired coagulation, angiogenesis and fibrinolysis plays an important role in the formation of CSDH.
Despite this dynamic hypothesis regarding the pathophysiology of CSDH, high-quality data supporting the use of DXM therapy as alternative treatment to surgery is scarce. Previous studies have shown favourable results of DXM as adjunctive to surgery in reducing mortality [24] and reoperation rate $[15,25,26]$. In current literature only four (non-randomised) studies evaluated the effect of corticosteroids in CSDH management as monotherapy compared to corticosteroids as an adjunctive to surgery or surgery alone [15]. In each study a different primary outcome measure was applied, of which only two used a validated outcome scale to assess functional outcome.

Of these two studies, the first study had a prospective design and evaluated 112 patients in four patient groups: DXM monotherapy, DXM in combination with surgery by BHC without additional drainage, surgery only and observation only [27]. This study revealed a favourable outcome, defined by a Glasgow Outcome Scale (GOS) score of 4-5 at 6 months, in $88 \%$ after DXM monotherapy. The reported success rate (GOS 4-5) for DXM therapy adjunctive to surgery was $91 \%$, compared to $77 \%$ after surgery alone and $50 \%$ after observation only. The second study described a retrospective evaluation in 122 patients in slightly different patient groups: initial DXM therapy, surgery alone by twist-drill mini-craniostomy, surgery alone by craniotomy and observation only [28]. A favourable outcome was expressed by a Markwalder Grading Scale (MGS) score of $0-2$ at discharge and was achieved in $73 \%$ after DXM monotherapy. In $25 \%$ of patients receiving initial DXM therapy, monotherapy failed and additional surgery was required in this group. In the primary surgical groups the reported success rates (MGS score 0-2) were 93 and 75\% after twist-drill mini-craniostomy and craniotomy, respectively, and for the observation only group $100 \%$.

In contrast, extensive research has been performed regarding the several operative techniques. Different surgical techniques can be applied, such as craniotomy, $\mathrm{BHC}$ or twist-drill craniostomy, with or without placement of a subdural drain. To date, no class I evidence exists to compare the various methods of haematoma evacuation. A recent large systematic review evaluated all 24 available RCTs regarding surgical treatment of CSDH. The only significant finding was a reduction in haematoma recurrence after postoperative subdural drainage based on eight RCTs [9]. In addition, one of the largest RCTs, performed in 215 symptomatic CSDH patients, showed that subdural drainage compared to no drainage not only lowered recurrence rate, but also reduced mortality and improved functional outcome at 6 months [10].

Overall, surgical techniques have been thoroughly demonstrated as effective therapy in the current literature for CSDH patients. DXM is showing promising results as an alternative treatment, but confirmation of these results is essential by means of RCTs. 


\section{Trial status}

This trial started on 1 September, 2016. The first patient was included in Medical Centre Haaglanden (HMC) The Hague and subsequently enrolment was started in Haga Teaching Hospital The Hague and Leiden University Medical Centre (LUMC) Leiden. The trial will start on 1 August 2018 at Erasmus Medical Centre (EMC) and Medisch Spectrum Twente (MST) and on 1 September 2018 at Isala Hospital Zwolle and Groningen University Medical Centre (UMCG). The study is open to additional participating neurosurgical centres.

\section{Additional files}

Additional file 1: Standard Protocol Items: Recommendations for Interventional Trials (SPIRIT) Checklist. (PDF 181 kb)

Additional file 2: Statistical analysis plan. (PDF $253 \mathrm{~kb}$ )

\section{Abbreviations}

BHC: Burr-hole craniostomy; CEA: Cost-effectiveness analyses; CSDH: Chronic subdural haematoma; CT: Computed tomography; DM: Diabetes mellitus; DSMB: Data Safety Monitoring Board; DXM: Dexamethasone; EMC: Erasmus University Medical Centre; GOS: Glasgow Outcome Scale; GOSE: Glasgow Outcome Scale-Extended; HMC: Haaglanden Medical Centre;

INR: International normalised ratio; LUMC: Leiden University Medical Centre; METC: Medical Ethics Committee; MGS: Markwalder Grading Scale; MRS: Modified Rankin Scale; MST: Medisch Spectrum Twente; NOAC: New oral anticoagulant; OD: Once a day; PCC: Prothrombin complex concentrate; QOLIBRI: Quality of Life in Brain Injury; RCT: Randomised controlled trial; SF-36: Short Form - 36 Health Survey; UMCG: University Medical Centre Groningen; VKA: Vitamin K antagonist; ZonMw: Netherlands Organisation for Health Research and Development

\section{Acknowledgements}

We want to thank R. Wolterbeek and Ewout Steyerberg (Department of Medical Statistics, Leiden University Medical Centre) and Suzanne Polinder (Department of Public Health Erasmus MC) for their support with the study design and analyses of the RCT and CEA. We also want to thank the local investigators for their effort in conducting the study in the participating centres.

\section{Funding}

Funding was received from Jacobus Stichting The Hague, the Research Bureau (Landsteiner Instituut) of the Haaglanden Medical Centre (HMC), ZonMw (Netherlands Organisation for Health Research and Development) grant (project number 843002824, 2017; EMC and MST) and the Erasmus MC (Mrace, project number 2016-16118; EMC). In a joined effort these are combined in the DECSA trial and the Dutch Subdural Haematoma Research Group was founded.

\section{Availability of data and materials}

The dataset generalised and analysed during the current study is available from the corresponding author on reasonable request.

\section{Protocol version}

This manuscript is based on the most recent protocol version, version 6 April 2018.

\section{Authors' contributions}

IPM designed the study, obtained funding, wrote the study protocol, is responsible for the implementation and organisation of the study in all participating centres, the conduct of the database and will perform the statistical analyses. WCP designed the study and is responsible for the local conduct of the study at LUMC. NAG designed the study and is responsible for the local conduct of the study at Haga Teaching Hospital. KJ designed the study and is responsible for the local conduct of the study at HMC. RD designed the study and is responsible for the local conduct of the study at EMC. HFL designed the study, obtained funding and is responsible for the statistical analyses and statistical analyses plan. W contributed to the design of the study. HMH designed the study and is responsible for the local conduct of the study at Isala Hospital. DCH wrote the study protocol and is, together with IPM, also responsible for the implementation and organisation of the study in all participating centres, the conduct of the database and will perform the statistical analyses. CMFD contributed to the design of the study. FK contributed to the design of the study. KHK contributed to the design of the study and is responsible for the local conduct of the study at MST. RW contributed to the design of the study and is, together with KJ, responsible for the local conduct of the study at HMC. NK contributed to the design of the study and is, together with WCP, responsible for the local conduct of the study at LUMC. RK contributed to the design of the study and is, together with WCP and NK, responsible for the local conduct of the study at LUMC. KL contributed to the design of the study and is, together with NAG, responsible for the local conduct of the study at Haga Teaching Hospital. JN contributed to the design of the study and is responsible for the local conduct of the study at UMCG. RJMG contributed to the design of the study and is, together with $J \mathrm{~N}$, responsible for the local conduct of the study at UMCG. BJ contributed to the design of the study and is, together with JN and RJMG, responsible for the local conduct of the study at UMCG. All authors read and approved the final version of the manuscript.

\section{Ethics approval and consent to participate}

The study received approval by the Medical Ethics Committee (METC Zuid West Holland). This trial has been registered in the European Union Clinical Trials Register (EUCTR) and is conducted in compliance with the European Union Clinical Trials Directive (2001/20/EC) and the principles of the Declaration of Helsinki (2013).

For eligible patients, written informed has to be obtained by their treating physician. If a patient is not capable to give written informed consent (i.e. due to altered consciousness or aphasia), the treating physician will ask the legal representative to provide written informed consent. Once the patient is capable of giving their own informed consent, the treating physician (at the ward or outpatient clinic) will ask the patient to provide written informed consent. If the patient withdraws their permission, the patient data will not be used for this study.

\section{Consent for publication}

By giving written informed consent, patients agree with the storage of data and publication of the study results.

\section{Competing interests}

The authors declare that they have no competing interests.

\section{Publisher's Note}

Springer Nature remains neutral with regard to jurisdictional claims in published maps and institutional affiliations.

\section{Author details}

${ }^{1}$ Department of Neurology and Neurosurgery, Haaglanden Medical Centre (HMC), Lijnbaan 32, 2512 VA The Hague, The Netherlands. ${ }^{2}$ Department of Neurology and Neurosurgery, Erasmus Medical Centre (EMC), Dr.

Molewaterplein 40, 3015 GD Rotterdam, The Netherlands. ${ }^{3}$ Department of Public Health, Erasmus Medical Centre (EMC), Dr. Molewaterplein 40, 3015 GD Rotterdam, The Netherlands. ${ }^{4}$ Department of Neurology and Neurosurgery, Leiden University Medical Centre (LUMC), Albinusdreef 2, 2333 ZA Leiden, The Netherlands. ${ }^{5}$ Department of Neurology and Neurosurgery, Haga Teaching Hospital, Els Borst-Eilersplein 275, 2545 AA The Hague, The Netherlands. ${ }^{6}$ Department of Neurosurgery, Medisch Spectrum Twente (MST), Koningsplein 1, 7512 KZ Enschede, The Netherlands. " Department of Neurology, Isala Hospital Zwolle, Dokter van Heesweg 2, 8025 AB Zwolle, The Netherlands. ${ }^{8}$ Department of Neurology and Neurosurgery, University of Groningen, University Medical Centre Groningen (UMCG), Hanzeplein 1, 9713 GZ Groningen, The Netherlands. 
Received: 30 December 2017 Accepted: 26 September 2018

Published online: 20 October 2018

\section{References}

1. Almenawer SA, Farrokhyar F, Hong C, Alhazzani W, Manoranjan B, Yarascavitch B, et al. Chronic subdural haematoma management: a systematic review and meta-analysis of 34829 patients. Ann Surg. 2014;259: 449-57.

2. Stichting Farmaceutische Kengetallen. Meer geneesmiddelen bij trombose. Pharmaceutisch Weekblad. 2008;143:41.

3. Ducruet AF, Grobelny BT, Zacharia BE, Hickman ZL, DeRosa PL, Andersen $\mathrm{KN}$, et al. The surgical management of chronic subdural haematoma. Neurosurg Rev. 2012;35:155-69.

4. Rust T, Kiemer N, Erasmus A. Chronic subdural haematomas and anticoagulation or anti-thrombotic therapy. J Clin Neurosci. 2006;13:823-7.

5. Miranda LB, Braxton E, Hobbs J, Quigley MR. Chronic subdural haematoma in the elderly: not a benign disease. J Neurosurg. 2011;114:72-6.

6. Asghar M, Adhiyaman V, Greenway MW, Bhowmick BK, Bates A. Chronic subdural haematoma in the elderly_a North Wales experience. J R Soc Med. 2002;95:290-2.

7. Kudo H, Kuwamura K, Izawa I, Sawa H, Tamaki N. Chronic subdural haematoma in elderly people: present status on Awaji Island and epidemiological prospect. Neurol Med Chir. 1992;32:207-9.

8. Kolias AG, Chari A, Santarius T, Hutchinson PJ. Chronic subdural haematoma: modern management and emerging therapies. Nat Rev Neurol. 2014:10:570-8.

9. Ivamoto HS, Lemos HP, Atallah AN. Surgical treatments for chronic subdural haematomas: a comprehensive systematic review. World Neurosurg. 2016; 86:399-418.

10. Santarius T, Kirkpatrick PJ, Ganesan D, Chia HL, Jalloh I, Smielewski P, et al. Use of drains versus no drains after burr-hole evacuation of chronic subdural haematoma: a randomised controlled trial. Lancet. 2009;374:1067-73

11. Liu W, Bakker NA, Groen RJM. Chronic subdural haematoma: a systematic review and meta-analysis of surgical procedures. J Neurosurg. 2014;121:665-73.

12. Weigel $R$, Schmiedek $P$, Krauss JK. Outcome of contemporary surgery for chronic subdural haematoma: evidence based review. J Neurol Neurosurg Psychiatry. 2003;74:937-43.

13. Drapkin AJ. Chronic subdural haematoma: pathophysiological basis for treatment. Br J Neurosurg. 1991;5:467-73.

14. Holl DC, Volovici V, Dirven CMF, Peul WC, Jellema K, van der Gaag NA, et al. Pathophysiology and targets for non-surgical therapy of chronic subdural haematoma: evolution from past to present to future. World Neurosurg. 2018;116:402-11.

15. Berhauser Pont LME, Dirven CMF, Dippel DWJ, Verweij BH, Dammers R. The role of corticosteroids in the management of chronic subdural haematoma: a systematic review. Eur J Neurol. 2012:19:1397-403.

16. Berkhemer OA, Fransen PSS, Beumer D, Van den Berg LA, Lingsma HF, Yoo $\mathrm{AJ}$, et al. A randomized trial of intraarterial treatment for acute ischemic stroke. N Engl J Med. 2015;372:11-20.

17. Markwalder T, Steinsiepe KF, Rohner M, Reichenbach W, Markwalder $H$. The course of chronic subdural haematomas after burr-hole craniostomy and closed-system drainage. J Neurosurg. 1981;55:390-6.

18. Ito H, Yamamoto S, Komai T, Mizukoshi H. Role of local hyperfibrinolysis in the etiology of chronic subdural haematoma. J Neurosurg. 1976;45:26-31.

19. Ito H, Komai T, Yamamoto S. Fibrinolytic enzyme in the lining walls of chronic subdural haematoma. J Neurosurg. 1978;48:197-200.

20. Labadie EL, Glover D. Local alterations of hemostatic-fibrinolytic mechanisms in reforming subdural haematomas. Neurology. 1975;25:669-75.

21. Labadie EL, Glover D. Physiopathogenesis of subdural haematomas: part II: inhibition of growth of experimental haematomas with dexamethasone. J Neurosurg. 1976;45:393-7.

22. Trappe A, Hafter $R$, Wendt $P$, Graeff $H$, Blümel $G$. Detection of fibrinolysis in chronic subdural haematoma. Neurochirurgia. 1986;29:78-82.

23. Edlmann E, Giorgi-Coll S, Whitfield PC, Carpenter KLH, Hutchinson PJ. Pathophysiology of chronic subdural haematoma: inflammation, angiogenesis and implications for pharmacotherapy. J Neuroinflammation. 2017;159:2037-44
24. Dran G, Berthier F, Fontaine D, Rasenrarijao D, Paquis P. Efficacité de la corticothérapie dans le traitement adjuvant des hématomes sous-duraux chroniques. Étude rétrospective sur 198 cas. Neurochirurgie. 2007:53:477-82.

25. Chan DYC, Sun TFD, Poon WS. Steroid for chronic subdural haematoma? A prospective phase IIB pilot randomized controlled trial on the use of dexamethasone with surgical drainage for the reduction of recurrence with surgical drainage for the reduction of recurrence with operation. Chin Neurosurg J. 2015

26. Qian Z, Yang D, Sun F, Sun Z. Risk factors for recurrence of chronic subdural haematoma after burr hole surgery: potential protective role of dexamethasone. Br J Neurosurg. 2017:31:84-8.

27. Sun TF, Boet R, Poon WS. Non-surgical primary treatment of chronic subdural haematoma: preliminary results of using dexamethasone. $\mathrm{Br}$ J Neurosurg. 2005;19:327-33.

28. Delgado-Lopez PD, Martin-Velasco V Castilla-Diez JM, Rodriquez-Salazar A, Galacho-Harriero AM, Fernandex-Arconada O. Dexamethasone treatment in chronic subdural haematoma. Neurocirugia (Astur). 2009;20:346-59.
Ready to submit your research? Choose BMC and benefit from:

- fast, convenient online submission

- thorough peer review by experienced researchers in your field

- rapid publication on acceptance

- support for research data, including large and complex data types

- gold Open Access which fosters wider collaboration and increased citations

- maximum visibility for your research: over $100 \mathrm{M}$ website views per year

At BMC, research is always in progress.

Learn more biomedcentral.com/submissions 